\title{
The Type I Generalized Half-Logistic Distribution Based on Upper Record Values
}

\author{
Devendra Kumar, ${ }^{1}$ Neetu Jain, ${ }^{2}$ and Shivani Gupta ${ }^{1}$ \\ ${ }^{1}$ Department of Statistics, Amity Institute of Applied Sciences, Amity University, Noida 201 303, India \\ ${ }^{2}$ Department of Statistics, PGDAV College, University of Delhi, Delhi 110007, India \\ Correspondence should be addressed to Devendra Kumar; devendrastats@gmail.com
}

Received 1 June 2015; Revised 9 July 2015; Accepted 26 July 2015

Academic Editor: Shein-chung Chow

Copyright @ 2015 Devendra Kumar et al. This is an open access article distributed under the Creative Commons Attribution License, which permits unrestricted use, distribution, and reproduction in any medium, provided the original work is properly cited.

\begin{abstract}
We consider the type I generalized half-logistic distribution and derive some new explicit expressions and recurrence relations for marginal and joint moment generating functions of upper record values. Here we show the computations for the first four moments and their variances. Next we show that results for record values of this distribution can be derived from our results as special cases. We obtain the characterization result of this distribution on using the recurrence relation for single moment and conditional expectation of upper record values. We obtain the maximum likelihood estimators of upper record values and their confidence intervals. Also, we compute the maximum likelihood estimates of the parameters of upper record values and their confidence intervals. At last, we present one real case data study to emphasize the results of this paper.
\end{abstract}

\section{Introduction}

The probability distribution which is a member of the family of logistic distribution is the half-logistic distribution with cumulative distribution function $(c d f)$ and probability density function $(p d f)$ that are given, respectively, by

$$
\begin{aligned}
& F(x)=\frac{e^{x}-1}{e^{x}+1}, \quad 0<x<\infty, \\
& f(x)=\frac{2 e^{x}}{\left(1+e^{x}\right)^{2}}, \quad 0<x<\infty .
\end{aligned}
$$

Balakrishnan [1] considered half-logistic probability models obtained as the models of the absolute value of the standard logistic models. Some key references about the half-logistic distribution include Balakrishnan and Aggarwala [2], Balakrishnan and Wong [3], and Balakrishnan and Chan [4]. Balakrishnan and Puthenpura [5] obtained the best linear unbiased estimators of location and scale parameters of the half-logistic distribution through linear functions of order statistics. Balakrishnan and Wong [6] obtained approximate maximum likelihood estimates for the location and scale parameters of the half-logistic distribution with type II rightcensoring. Torabi and Bagheri [7] gave the estimators of parameters for the extended generalized half-logistic distribution based on complete and censored data.

Record values are found in many situations of daily life as well as in many statistical applications. Often we are interested in observing new records and in recording them, for example, Olympic records or world records in sport. Record values are also used in reliability theory. Moreover, these statistics are closely connected with the occurrence times of some corresponding nonhomogeneous Poisson process used in shock models. The statistical study of record values started with Chandler [8]; he formulated the theory of record values as a model for successive extremes in a sequence of independently and identically random variables. Feller [9] gave some examples of record values with respect to gambling problems. Resnick [10] discussed the asymptotic theory of records. Theory of record values and its distributional properties have been extensively studied in the literature; for example, Ahsanullah [11], Arnold et al. [12, 13], Nevzorov [14], and Kamps [15] can be seen for reviews on various developments in the area of records. 
We will now consider the situations in which the record values (e.g., successive largest insurance claims in nonlife insurance, highest water-levels, or highest temperatures) themselves are viewed as "outliers" and hence the second or third largest values are of special interest. Insurance claims in some nonlife insurance can be used as one of the examples. Observing successive $k$ th largest values in a sequence, Dziubdziela and Kopocinski [16] proposed the following model of $k$ th record values, where $k$ is some positive integer.

Let $X_{1}, X_{2}, \ldots$ be a sequence of identically independently distributed (iid) random variables with $p d f f(x)$ and cdf $F(x)$. Let $X_{j: n}$ denote the $j$ th order statistic of a sample $\left(X_{1}, X_{2}, \ldots, X_{n}\right)$. For a fixed $k \geq 1$ we define the sequence $U_{1}^{(k)}, U_{2}^{(k)}, \ldots$ of $k$ th upper record times of $X_{1}, X_{2}, \ldots$ as follows:

$$
\begin{aligned}
& U_{1}^{(k)}=1, \\
& U_{n+1}^{(k)}=\min \left\{j>U_{n}^{(k)}: X_{j: j+k-1}>X_{U_{n}^{(k)}: U_{n}^{(k)}+k-1}\right\} \\
& n=1,2, \ldots .
\end{aligned}
$$

The sequence $\left\{Y_{n}^{(k)}, n \geq 1\right\}$, where $Y_{n}^{(k)}=X_{U_{n}^{(k)}}$, is called the sequences of $k$ th upper record values of the sequence $\left\{X_{n}, n \geq\right.$ $1\}$. For convenience, we define $Y_{0}^{(k)}=0$. Note that for $k=1$ we have $Y_{n}^{(1)}=X_{(n)}, n \geq 1$, which are record values of $\left\{X_{n}, n \geq 1\right\}$ [11].

Let $\left\{Y_{n}^{(k)}, n \geq 1\right\}$ be the sequence of $k$ th upper record values. Then the $p d f$ of $Y_{n}^{(k)}, n \geq 1$, is as follows:

$$
f_{Y_{n}^{(k)}}(x)=\frac{k^{n}}{(n-1) !}[-\ln (\bar{F}(x))]^{n-1}[\bar{F}(x)]^{k-1} f(x) .
$$

Also the joint density function of $Y_{m}^{(k)}$ and $Y_{n}^{(k)},(1 \leq m<n)$, $n=2,3, \ldots$, as discussed by Grudzień [17] is given by

$$
\begin{aligned}
& f_{Y_{m}^{(k)}, Y_{n}^{(k)}}(x, y) \\
& \quad=\frac{k^{n}}{(m-1) !(n-m-1) !}[-\ln (\bar{F}(x))]^{m-1} \\
& \quad \cdot[-\ln (\bar{F}(y))+\ln (\bar{F}(x))]^{n-m-1}[\bar{F}(y)]^{k-1} \frac{f(x)}{\bar{F}(x)} \\
& \quad \cdot f(y), \quad x<y,
\end{aligned}
$$

where

$$
\bar{F}(x)=1-F(x) .
$$

Kumar [18] established recurrence relations for moment generating function of $k$ th record values from generalized logistic distribution. Recurrence relations for moment generating function of record values from Pareto, Gumble, power function, and extreme value distributions are derived by Ahsanullah and Raqab [19] and Raqab and Ahsanullah [20, 21], respectively. Recurrence relations for single and product moments of $k$ th record values from Weibull, Pareto, generalized Pareto, Burr, exponential, and Gumble distribution are derived by Pawlas and Szynal [22-24]. Sultan [25] established recurrence relations for moments of $k$ record values from modified Weibull distribution. Kumar [26] and Kumar and Kulshrestha [27] have established recurrence relations for moments of $k$ th record values from exponentiated loglogistic and generalized Pareto distributions, respectively.

In the next section, we present some explicit expressions and recurrence relations for marginal moment generating functions of $k$ th upper record values from type I generalized half-logistic distribution and results for record values are deduced as special case. The obtained relations were used to compute mean and variance, upper record values. In Section 3, we discuss joint moment generating function of $k$ th upper record values from type I generalized half-logistic distribution and results for upper record values are deduced as special case. In Section 4, we present a characterization of this distribution by using recurrence relation for single moment and conditional expectation of record values. In Section 5, we obtain maximum likelihood estimators of $k$ th upper record values from type I generalized half-logistic distribution and the confidence intervals for their estimation. Section 6 consists of simulation study based on the maximum likelihood estimates of the parameters based on upper record values of true values of parameters. In Section 7 , the analysis of one real data example is provided to illustrate the performance of maximum likelihood estimates of type I generalized half-logistic distribution. Some final comments in Section 8 conclude the paper.

\section{Type I Generalized Half-Logistic Distribution}

Olapade [28] proposed $c d f$ of three-parameter type I generalized half-logistic distribution and obtained some basic properties such as moments, median, and mode and also estimated its parameters by maximum likelihood approach. The three-parameter type I generalized half-logistic distribution has the $c d f$

$$
\begin{aligned}
& F(x ; \theta, \sigma, \mu)=1-\frac{2^{\theta}}{\left[1+e^{(x-\mu) / \sigma}\right]^{\theta}}, \\
& \quad \mu \leq x<\infty, \theta, \sigma, \mu>0 .
\end{aligned}
$$

Therefore, type I generalized half-logistic distribution has $p d f$

$$
\begin{aligned}
f(x ; \theta, \sigma, \mu)=\frac{2^{\theta} \theta e^{(x-\mu) / \sigma}}{\sigma\left[1+e^{(x-\mu) / \sigma}\right]^{\theta+1}}, & \\
& \mu \leq x<\infty, \theta, \sigma, \mu>0 .
\end{aligned}
$$

Here $\theta$ is the shape parameter and $\mu$ and $\sigma$ are the location and scale parameter, respectively. Plotted are the probability density function (Figure 1), hazard rate function (Figure 2), and survival function (Figure 3 ) for some values of parameters.

2.1. Hazard Rate Function. This function is used in analysis of time relating to the event and describes the current chance 

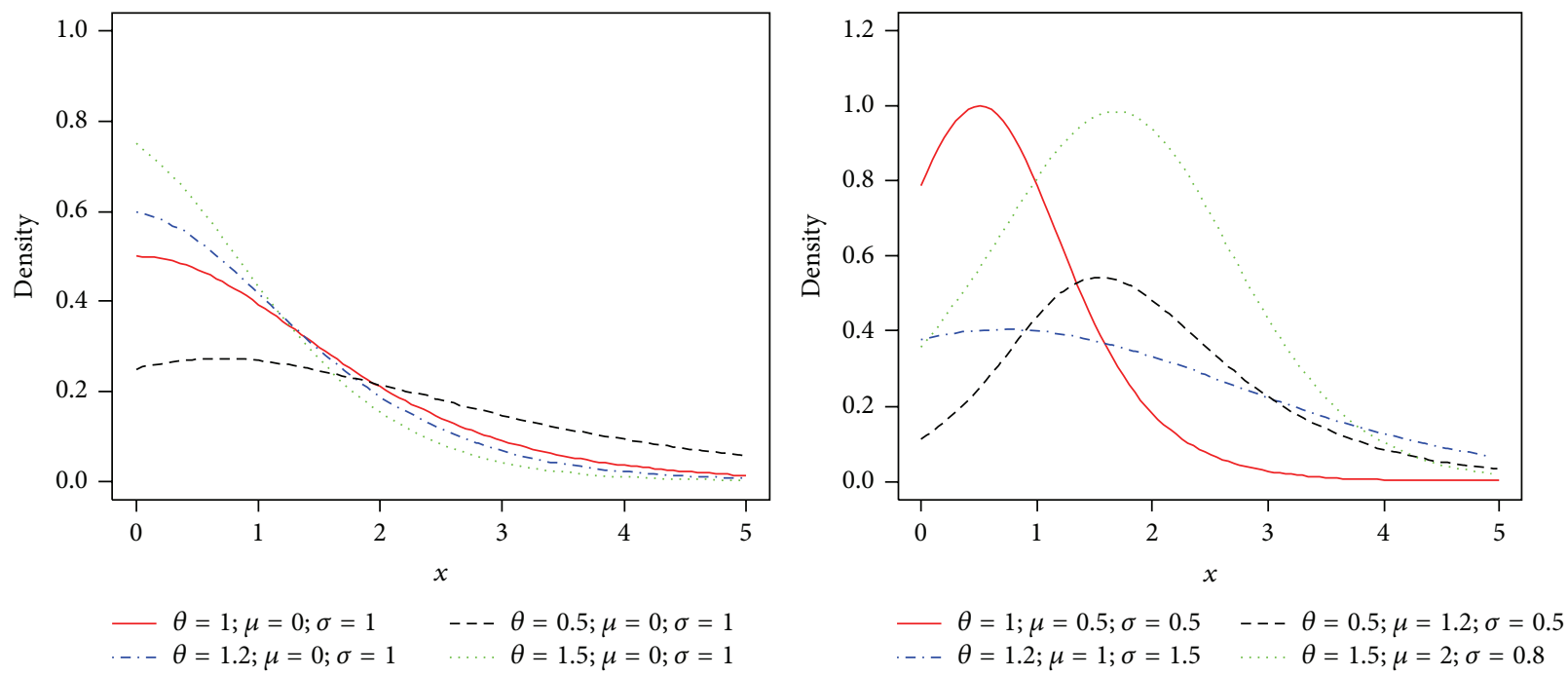

Figure 1: Probability density function of the type I generalized half-logistic distribution for the indicated values of $\theta, \sigma$, and $\mu$.
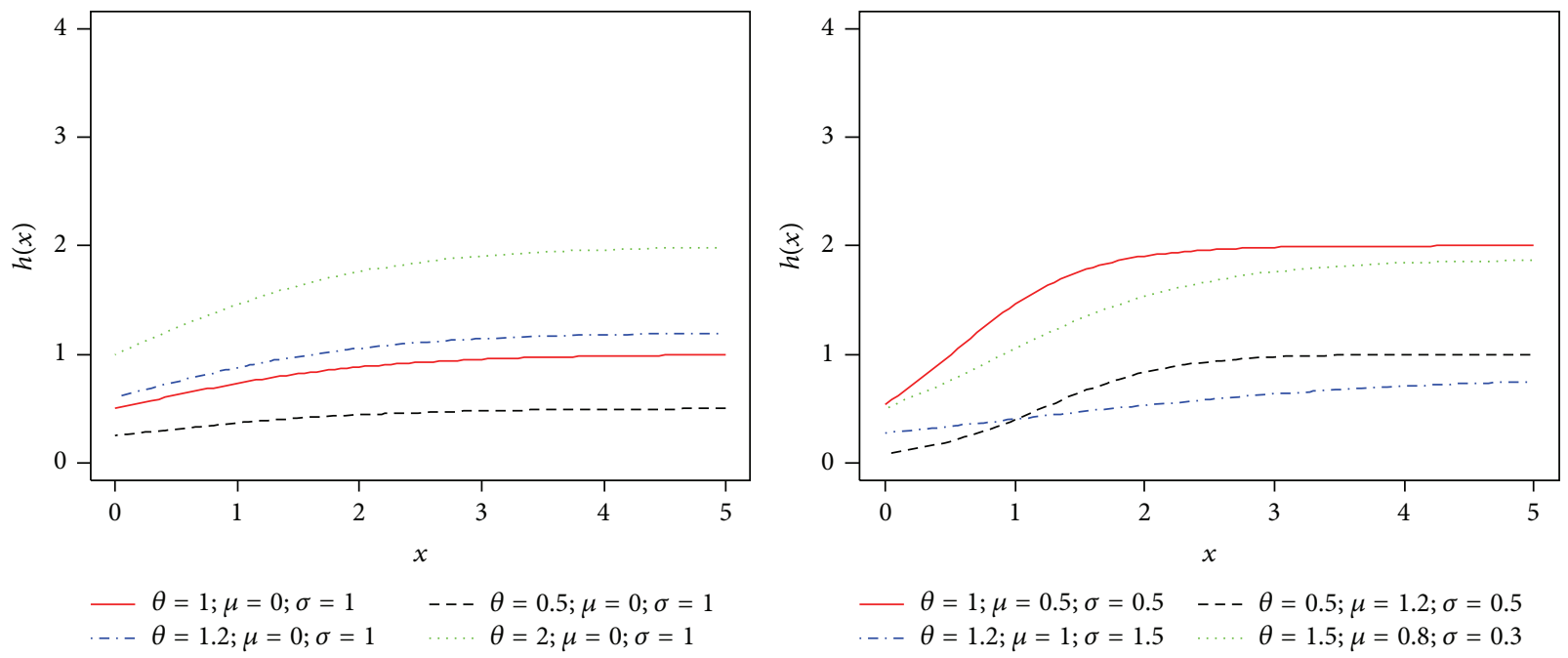

FIGURE 2: Hazard rate function of the type I generalized half-logistic distribution for the indicated values of $\theta, \sigma$, and $\mu$.
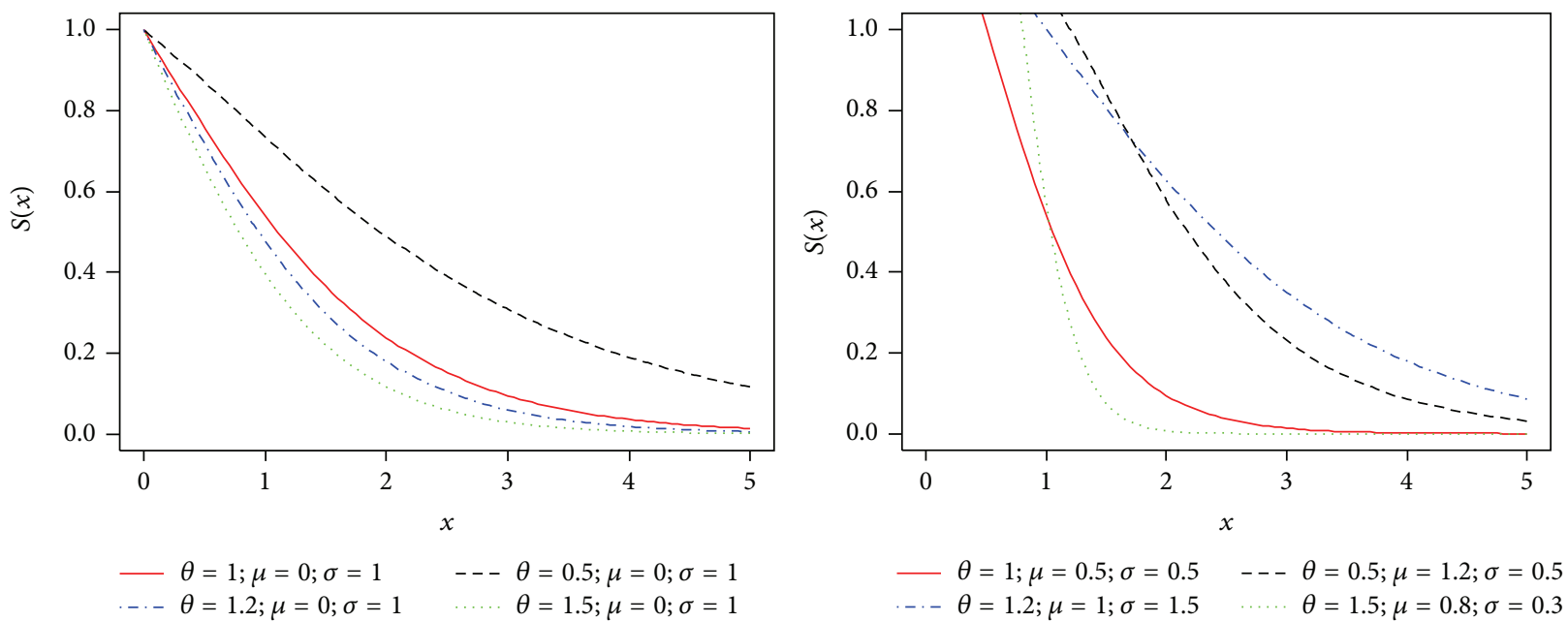

FIGURE 3: Survival function of the type I generalized half-logistic distribution for the indicated values of $\theta, \sigma$, and $\mu$. 
of failure for the population that has not been failed yet. Hazard rate function plays an important role in reliability analysis, survival analysis, and demography and in defining and formulating a model when dealing with lifetime data.

For the type I generalized half-logistic distribution, hazard rate function takes the form

$$
h(x)=\frac{\theta}{\sigma\left[1+e^{-(x-\mu) / \sigma}\right]}, \quad 0 \leq x<\infty, \theta, \sigma, \mu>0 .
$$

2.2. Survival Function. In engineering science, it is called reliability analysis. In fact the survival function is the probability of failure by time $x$, where $x$ represents survival time. We use survival function to predict quantiles of the survival time. Survival function is given by

$$
S(x)=\frac{2^{\theta}}{\left[1+e^{(x-\mu) / \sigma}\right]^{\theta}}, \quad 0 \leq x<\infty, \theta, \sigma, \mu>0 .
$$

We assume through this study, without loss of generality, that $\mu=0$ and $\sigma=1$, in which case the $p d f$ and $c d f$ are, respectively, reduced to

$$
f(x ; \theta)=\frac{2^{\theta} \theta e^{x}}{\left(1+e^{x}\right)^{\theta+1}}, \quad 0 \leq x<\infty, \theta>0,
$$

and the corresponding $c d f$ is

$$
F(x ; \theta)=1-\frac{2^{\theta}}{\left(1+e^{x}\right)^{\theta}}, \quad 0 \leq x<\infty, \theta>0 .
$$

If $\theta=1$, then the type I generalized half-logistic distribution reduces to the half-logistic distribution.

A recurrence relation for single and product moments of upper record values from the type I generalized half-logistic distribution is obtained by making use of the following differential equation (obtained from (10) and (11)):

$$
\theta \bar{F}(x)=\left(1+e^{-x}\right) f(x) .
$$

Let us denote the marginal moment generating functions of $X_{U(n): k}$ by $M_{X_{U(n): k}}(t)$ and its $j$ th derivative by $M_{X_{U(n)}: k}^{(j)}(t)$. Similarly, let $M_{X_{U(m, n) k}}\left(t_{1}, t_{2}\right)$ denote the joint moment generating function of $X_{U(m): k}$ and $X_{U(n): k}$ and its $(i, j)$ th partial derivatives by $M_{X_{U(m, n): k}}^{(i, j)}\left(t_{1}, t_{2}\right)$ with respect to $t_{1}$ and $t_{2}$, respectively.

We will first establish the explicit expressions for marginal moment generating function of $k$ th upper record values $M_{X_{U(n): k}}(t)$ by the following theorem.

Theorem 1. For distribution as given in (11) and $k \geq 1, n \geq 1$, $r=1,2, \ldots$,

$$
\begin{aligned}
& M_{X_{U(n): k}}(t) \\
& \quad=\sum_{p=0}^{\infty}(-1)^{p} \frac{\Gamma(t+1) 2^{t-p}}{p ! \Gamma(t+1-p)(1-(p-t) / \theta k)^{n}} .
\end{aligned}
$$

Proof. From (3), we have

$$
\begin{aligned}
& M_{X_{U(n): k}}(t)=\frac{k^{n}}{(n-1) !} \\
& \cdot \int_{-\infty}^{\infty} e^{t x}[\bar{F}(x)]^{k-1}[-\ln (\bar{F}(x))]^{n-1} f(x) d x .
\end{aligned}
$$

By making use of the transformation $z=[\bar{F}(x)]^{1 / \theta}$ in (14), we get the desired result as (13).

Remark 2. Setting $k=1$ in (13) we deduce the explicit expression of marginal moment generating function of upper record values from the type I generalized half-logistic distribution.

Recurrence relations for marginal moment generating function of $k$ th upper record values from $c d f$ (11) are derived in the following theorem.

Theorem 3. For a positive integer $k \geq 1$ and for $n \geq 1$ and $r=0,1,2, \ldots$,

$$
\left(1-\frac{t}{\theta k}\right) M_{X_{U(n): k}}^{(j)}(t)=M_{X_{U(n-1): k}}^{(j)}(t)+\frac{t}{\theta k} M_{X_{U(n): k}}^{(j-1)}(t) .
$$

Proof. From (3), we have

$$
\begin{aligned}
& M_{X_{U(n): k}}(t)=\frac{k^{n}}{(n-1) !} \\
& \cdot \int_{-\infty}^{\infty} e^{t x}[\bar{F}(x)]^{k-1}[-\ln (\bar{F}(x))]^{n-1} f(x) d x .
\end{aligned}
$$

Integrating by parts, taking $[\bar{F}(x)]^{k-1} f(x)$ as the part to be integrated and the rest of the integrand for differentiation, we get

$$
\begin{aligned}
& M_{X_{U(n): k}}(t) \\
& =M_{X_{U(n-1): k}}(t) \\
& \quad+\frac{t k^{n}}{k(n-1) !} \int_{-\infty}^{\infty} e^{t x}[\bar{F}(x)]^{k}[-\ln (\bar{F}(x))]^{n-1} d x .
\end{aligned}
$$

The constant of integration vanishes since the integral considered in (16) is a definite integral. On using (12), we obtain

$$
\begin{aligned}
& M_{X_{U(n): k}}(t)=M_{X_{U(n-1): k}}(t) \\
& +\frac{t k^{n}}{\theta k(n-1) !}\left\{\int_{0}^{\infty} e^{(t-1) x}[\bar{F}(x)]^{k-1}\right. \\
& \cdot[-\ln (\bar{F}(x))]^{n-1} f(x) d x \\
& \left.+\int_{0}^{\infty} e^{t x}[\bar{F}(x)]^{k-1}[-\ln (\bar{F}(x))]^{n-1} f(x) d x\right\} \\
& M_{X_{U(n): k}}(t)=M_{X_{U(n-1): k}}(t)+\frac{t}{\theta k} M_{X_{U(n): k}}(t)+\frac{t}{\theta k} \\
& \cdot M_{X_{U(n): k}}(t-1) .
\end{aligned}
$$


Differentiating both sides of (18) $j$ times with respect to $t$, we get

$$
\begin{aligned}
M_{X_{U(n): k}}^{(j)}(t)= & M_{X_{U(n-1): k}}^{(j)}(t)+\frac{t}{\theta k} M_{X_{U(n): k}}^{(j)}(t) \\
& +\frac{j}{\theta k} M_{X_{U(n): k}}^{(j-1)}(t)+\frac{t}{\theta k} M_{X_{U(n): k}}^{(j)}(t-1) \\
& -\frac{j}{\theta k} M_{X_{U(n): k}}^{(j-1)}(t-1)
\end{aligned}
$$

The recurrence relation in (15) is derived simply by rewriting the above equation.

By differentiating both sides of (15) with respect to $t$ and then setting $t=0$, we obtain the recurrence relations for single moment of $k$ th upper record values from type I generalized half-logistic distribution in the form

$$
\begin{aligned}
E\left(X_{U(n): k}^{j}\right)= & E\left(X_{U(n-1): k}^{j}\right) \\
& +\frac{j}{\theta k}\left[E\left(X_{U(n): k}^{j-1}\right)+E\left(\phi\left(X_{U(n): k}\right)\right)\right],
\end{aligned}
$$

where

$$
\phi(x)=x^{j-1} e^{-x} .
$$

Remark 4. Setting $k=1$ in (15), we deduce the recurrence relation for marginal moment generating function of upper record values from the type I generalized half-logistic distribution.

The mean and variances of upper record values of a type I generalized half-logistic distribution for different values of $\theta$ are calculated in Tables 1 and 2, respectively.

It appears from the results that the mean of upper record values increases with size being increased. In addition, depending on the values of $\theta$ the mean of upper record values of the distribution can be greater than its variance.

\section{Relations for Joint Moment Generating Functions}

The explicit expression for the joint moment generating functions of $k$ th upper record values is derived by the following theorem.

Theorem 5. For the distribution as given in (10), $1 \leq m \leq n-2$, $r, s=1,2, \ldots$,

$$
\begin{aligned}
& M_{X_{U(m, n): k}}\left(t_{1}, t_{2}\right)=(\theta k)^{n} \\
& \cdot \sum_{p=0}^{\infty} \sum_{q=0}^{\infty} \frac{(-1)^{p+q} 2^{p+q-\left(t_{1}+t_{2}\right)} \Gamma\left(t_{2}+1\right)}{p ! q ! \Gamma\left(t_{2}+1-p\right) \Gamma\left(t_{1}+1-q\right)} \\
& \cdot \frac{\Gamma(t+1)}{\left[\theta k+p+t_{2}\right]^{n-m}\left[\theta k+p-q-t_{1}-t_{2}\right]^{m}} .
\end{aligned}
$$

TABLE 1: Mean of upper records.

\begin{tabular}{ccccc}
\hline$n$ & $\theta=1$ & $\theta=2$ & $\theta=3$ & $\theta=4$ \\
\hline 1 & 1.3862 & 0.7725 & 0.5451 & 0.4237 \\
2 & 2.5507 & 1.4305 & 1.0189 & 0.7982 \\
3 & 3.6252 & 2.0259 & 1.4483 & 1.1396 \\
4 & 4.6601 & 2.5852 & 1.8487 & 1.4579 \\
5 & 5.6769 & 3.1228 & 2.2298 & 1.7597 \\
\hline
\end{tabular}

TABLE 2: Variance of upper records.

\begin{tabular}{cccc}
\hline$n$ & $\theta=2$ & $\theta=3$ & $\theta=4$ \\
\hline 1 & 0.43784 & 0.22676 & 0.14147 \\
2 & 0.71407 & 0.37174 & 0.23488 \\
3 & 0.94243 & 0.48383 & 0.30581 \\
4 & 1.15894 & 0.58331 & 0.36593 \\
5 & 1.37612 & 0.67789 & 0.42086 \\
\hline
\end{tabular}

Proof. From (4), we have

$$
\begin{gathered}
M_{X_{U(m, n): k}}\left(t_{1}, t_{2}\right)=\frac{k^{n}}{(m-1) !(n-m-1) !} \\
\cdot \int_{-\infty}^{\infty} e^{t_{1} x}[-\ln (\bar{F}(x))]^{m-1} \frac{f(x)}{\bar{F}(x)} G(x) d x,
\end{gathered}
$$

where

$$
\begin{aligned}
& G(x)=\int_{x}^{\infty} e^{t_{2} y}[\ln (\bar{F}(x))-\ln (\bar{F}(y))]^{n-m-1} \\
& \cdot[\bar{F}(y)]^{k-1} f(y) d y .
\end{aligned}
$$

By setting $w=-\ln \bar{F}(y)+\ln \bar{F}(x)$ and simplifying the resulting expression we get

$$
\begin{aligned}
& G(x) \\
& =\sum_{p=0}^{\infty}(-1)^{P} \frac{2^{t-p} \Gamma\left(t_{2}+1\right) \Gamma(n-m)[\bar{F}(x)]^{k+\left(p-t_{2}\right) \theta}}{p ! \Gamma\left(t_{2}+1-p\right)((\theta k+p+t) / \theta)^{n-m}} .
\end{aligned}
$$

Substituting the value of $G(x)$ in equation (23) and simplifying we get result given in (22).

Remark 6. Setting $k=1$ in (22) we deduce the explicit expression for joint moment generating functions of upper record values for the type I generalized half-logistic distribution.

Recurrence relations for joint moment generating functions of $k$ th upper record values $c d f$ (6) can be derived in the following theorem.

Theorem 7. For $1 \leq m \leq n-2$ and $r, s=0,1,2, \ldots$,

$$
\begin{aligned}
& \left(1-\frac{t_{2}}{\theta k}\right) M_{X_{U(m, n): k}}^{(i, j)}\left(t_{1}, t_{2}\right) \\
& \quad=M_{X_{U(m, n-1): k}}^{(i, j)}\left(t_{1}, t_{2}\right)+\frac{t_{2}}{\theta k} M_{X_{U(m, n): k}}^{(i, j-1)}\left(t_{1}, t_{2}\right) .
\end{aligned}
$$


Proof. From (4) for $1 \leq m \leq n-1, r, s=0,1,2, \ldots$,

$$
\begin{array}{r}
M_{X_{U(m, n): k}}\left(t_{1}, t_{2}\right)=\frac{k^{n}}{(m-1) !(n-m-1) !} \\
\cdot \int_{-\infty}^{\infty}[-\ln (\bar{F}(x))]^{m-1} \frac{f(x)}{\bar{F}(x)} I(x) d x,
\end{array}
$$

where

$$
\begin{aligned}
& I(x)=\int_{x}^{\infty} e^{t_{1} x+t_{2} y}[\ln (\bar{F}(x))-\ln (\bar{F}(y))]^{n-m-1} \\
& \cdot[\bar{F}(y)]^{k-1} f(y) d y .
\end{aligned}
$$

Integrating $I(x)$ by parts, taking $[\bar{F}(y)]^{k-1} f(y)$ for integration and the rest of the integrand for differentiation, and substituting the resulting expression in (27), we get

$$
\begin{aligned}
& M_{X_{U(m, n): k}}\left(t_{1}, t_{2}\right)=M_{X_{U(m, n-1): k}}\left(t_{1}, t_{2}\right) \\
& +\frac{t_{2} k^{n}}{k(m-1) !(n-m-1) !} \\
& \cdot \int_{-\infty}^{\infty} \int_{x}^{\infty} e^{t_{1} x+t_{2} y}[-\ln (\bar{F}(x))]^{m-1} \\
& \cdot[\ln (\bar{F}(x))-\ln (\bar{F}(y))]^{n-m-1}[\bar{F}(y)]^{k} \\
& \cdot \frac{f(x)}{\bar{F}(x)} d y d x .
\end{aligned}
$$

The constant of integration vanishes since the integral in $I(x)$ is a definite integral. On using the relation (10), we obtain

$$
\begin{aligned}
& M_{X_{U(m, n): k}}\left(t_{1}, t_{2}\right)=M_{X_{U(m, n-1): k}}\left(t_{1}, t_{2}\right)+\frac{t_{2} k^{n}}{\theta k(m-1) !(n-m-1) !}\left\{\int_{0}^{\infty} \int_{x}^{\infty} e^{t_{1} x+\left(t_{2}-1\right) y}[-\ln (\bar{F}(x))]^{m-1} f(x)\right. \\
& \cdot[\ln (\bar{F}(x))-\ln (\bar{F}(y))]^{n-m-1}[\bar{F}(y)]^{k-1} \frac{f(y)}{\bar{F}(x)} d y d x+\int_{0}^{\infty} \int_{x}^{\infty} e^{t_{1} x+t_{2} y}[-\ln (\bar{F}(x))]^{m-1} f(x) \\
& \left.\cdot[\ln (\bar{F}(x))-\ln (\bar{F}(y))]^{n-m-1}[\bar{F}(y)]^{k-1} \frac{f(y)}{\bar{F}(x)} d y d x\right\}, \\
& M_{X_{U(m, n): k}}\left(t_{1}, t_{2}\right)=M_{X_{U(m, n-1): k}}\left(t_{1}, t_{2}\right)+\frac{t_{2}}{\theta k} M_{X_{U(m, n): k}}\left(t_{1}, t_{2}\right)+\frac{t_{2}}{\theta k} M_{X_{U(m, n): k}}\left(t_{1}, t_{2}-1\right) .
\end{aligned}
$$

Differentiating both sides of (30) $i$ times with respect to $t_{1}$ and then $j$ times with respect to $t_{2}$, we get

$$
\begin{aligned}
& M_{X_{U(m, n): k}}^{(i, j)}\left(t_{1}, t_{2}\right)=M_{X_{U(m, n-1): k}}^{(i, j)}\left(t_{1}, t_{2}\right) \\
& +\frac{t_{2}}{\theta k} M_{X_{U(m, n): k}}^{(i, j)}\left(t_{1}, t_{2}\right) \\
& +\frac{j}{\theta k} M_{X_{U(m, n): k}}^{(i, j-1)}\left(t_{1}, t_{2}\right) \\
& +\frac{t_{2}}{\theta k} M_{X_{U(m, n): k}}^{(i, j)}\left(t_{1}, t_{2}-1\right) \\
& +\frac{j}{\theta k} M_{X_{L(m, n): k}}^{(i, j-1)}\left(t_{1}, t_{2}-1\right)
\end{aligned}
$$

and hence the result is given in (26).

By differentiating both sides of (30) with respect to $t_{1}$, $t_{2}$ and then setting $t_{1}=t_{2}=0$, we obtain the recurrence relations for product moments of $k$ th upper record values from type I generalized half-logistic distribution in the form

$$
\begin{aligned}
E( & \left.X_{U(m, n): k}^{(i, j)}\right) \\
= & E\left(X_{U(m, n-1): k}^{(i, j)}\right) \\
& +\frac{j}{\theta k}\left\{E\left(X_{U(m, n): k}^{(i, j-1)}\right)+E\left(\phi\left(X_{U(m, n): k}\right)\right)\right\},
\end{aligned}
$$

where

$$
\phi(x)=x^{i} y^{j-1} e^{-y}
$$

Remark 8. Setting $k=1$ in (26), we deduce the recurrence relation for joint moment generating functions of upper record values for the type I generalized half-logistic distribution. 


\section{Characterizations}

This section contains characterizations of type I generalized half-logistic distribution based on recurrence relation of marginal moment generating functions of $k$ th upper record values and conditional expectation of upper record values.

Let $L(a, b)$ stand for the space of all integrable functions on $(a, b)$. A sequence $\left(f_{n}\right) \subset L(a, b)$ is called complete on $L(a, b)$ if, for all functions $g \in L(a, b)$, the condition

$$
\int_{a}^{b} g(x) f_{n}(x) d x=0, \quad n \in N
$$

implies $g(x)=0$ a.e. on $(a, b)$. We start with the following result of Lin [29].

Proposition 9. Let $n_{0}$ be any fixed nonnegative integer, $-\infty \leq$ $a<b \leq \infty$, and $g(x) \geq 0$ an absolutely continuous function with $g^{\prime}(x) \neq 0$ a.e. on $(a, b)$. Then the sequence of functions $\left\{(g(x))^{n} e^{-g(x)}, n \geq n_{0}\right\}$ is complete in $L(a, b)$ iff $g(x)$ is strictly monotone on $(a, b)$.

Using the above proposition we get a stronger version of Theorem 1.

Theorem 10. Let $X$ be a nonnegative random variable having an absolutely continuous distribution function $F(x)$ with $F(0)=0$ and $0<F(x)<1$ for all $x>0$ :

$$
\left(1-\frac{t}{\theta k}\right) M_{X_{U(n): k}}^{(j)}(t)=M_{X_{U(n-1): k}}^{(j)}(t)+\frac{t}{\theta k} M_{X_{U(n): k}}^{(j-1)}(t)
$$

if and only if

$$
F(x)=1-\frac{2^{\theta}}{\left(1+e^{x}\right)^{\theta}}, \quad 0 \leq x<\infty, \theta>0 .
$$

Proof. The necessary part follows immediately from (15). On the other hand if the recurrence relation in (35) is satisfied, then on using (3), we have

$$
\begin{aligned}
& \frac{k^{n}}{(n-1) !} \int_{0}^{\infty} e^{t x}[\bar{F}(x)]^{k-1}[-\ln (\bar{F}(x))]^{n-1} f(x) d x \\
& \quad=\frac{t(n-1) k^{n}}{k(n-1) !} \\
& \cdot \int_{0}^{\infty} e^{t x}[\bar{F}(x)]^{k-1}[-\ln (\bar{F}(x))]^{n-2} f(x) d x \\
& +\frac{t k^{n}}{\theta k(n-1) !} \\
& \cdot \int_{0}^{\infty} e^{(t-1) x}[\bar{F}(x)]^{k-1}[-\ln (\bar{F}(x))]^{n-1} f(x) d x \\
& +\frac{t k^{n}}{\theta k(n-1) !} \\
& \cdot \int_{0}^{\infty} e^{t x}[\bar{F}(x)]^{k-1}[-\ln (\bar{F}(x))]^{n-1} f(x) d x .
\end{aligned}
$$

Integrating the first integral on the right hand side of (37), by parts, we get

$$
\begin{gathered}
\frac{t k^{n}}{k(n-1) !} \int_{0}^{\infty} e^{t x}[\bar{F}(x)]^{k-1}[-\ln (\bar{F}(x))]^{n-1} \\
\cdot\left(\bar{F}(x)-\frac{e^{-x}}{\theta} f(x)+\frac{f(x)}{\theta}\right) d x=0 .
\end{gathered}
$$

It now follows from Proposition 9 that

$$
\theta \bar{F}(x)=\left(e^{-x}+1\right) f(x)
$$

which proves that

$$
F(x)=1-\frac{2^{\theta}}{\left(1+e^{x}\right)^{\theta}}, \quad 0 \leq x<\infty, \theta>0 .
$$

Let $\left\{X_{n}, n \geq 1\right\}$ be a sequence of $i$ id. continuous random variables with $c d f F(x)$ and $p d f f(x)$. Let $Y_{U(n)}$ be the $n$th upper record value; then the conditional $p d f$ of $Y_{U(n)}$ given $Y_{U(m)}=x, 1 \leq m<n$, in view of (3) and (4), for $k=1$ is

$$
\begin{aligned}
f & \left(Y_{U(n)} \mid Y_{U(m)}=x\right) \\
& =\frac{1}{(n-m-1) !}[-\ln \bar{F}(y)+\ln \bar{F}(x)]^{n-m-1} \frac{f(y)}{\bar{F}(x)} .
\end{aligned}
$$

Theorem 11. Let $X$ be an absolutely continuous $r v$ with cdf $F(x)$ and pdf $f(x)$ on the support $(-\infty, \infty)$; then, for $m<n$,

$$
\begin{aligned}
& E\left[e^{t X_{U(n)}} \mid X_{U(m)}=x\right] \\
& \quad=\sum_{p=0}^{t}(-1)^{p+t}\left(\begin{array}{l}
t \\
p
\end{array}\right)\left(1+e^{x}\right)^{p}\left(\frac{\theta}{\theta-p}\right)^{n-m}
\end{aligned}
$$

if and only if

$$
F(x)=1-\frac{2^{\theta}}{\left(1+e^{x}\right)^{\theta}}, \quad 0 \leq x<\infty, \theta>0 .
$$

Proof. From (41), we have

$$
\begin{gathered}
E\left[e^{t X_{U(n)}} \mid X_{U(m)}=x\right]=\frac{1}{(n-m-1) !} \\
\cdot \int_{x}^{\infty} e^{t y}\left[\ln \left(\frac{\bar{F}(x)}{\bar{F}(y)}\right)\right]^{n-m-1} \frac{f(y)}{\bar{F}(x)} d y .
\end{gathered}
$$

By setting $u=\ln (\bar{F}(x) / \bar{F}(y))$ from (4) in (44), we obtain

$$
\begin{gathered}
E\left[e^{t X_{U(n)}} \mid X_{U(m)}=x\right]=\frac{1}{(n-m-1) !} \sum_{p=0}^{t}(-1)^{p+t} \\
\cdot\left(\begin{array}{l}
t \\
p
\end{array}\right)\left(1+e^{x}\right)^{p} \int_{0}^{\infty} e^{-(1-p / \theta) u} u^{n-m-1} d u .
\end{gathered}
$$


Simplifying the above expression, we derive the relation given in (42).

To prove sufficient part, we have from (41) and (42) that

$$
\begin{aligned}
& \frac{1}{(n-m-1) !} \\
& \cdot \int_{x}^{\infty} e^{t y}[-\ln \bar{F}(y)+\ln \bar{F}(x)]^{n-m-1} f(y) \\
& =\bar{F}(x) H_{m}(x),
\end{aligned}
$$

where

$$
H_{m}(x)=\sum_{p=0}^{t}(-1)^{p+t}\left(\begin{array}{l}
t \\
p
\end{array}\right)\left(1+e^{x}\right)^{p}\left(\frac{\theta}{\theta-p}\right)^{n-m} .
$$

Differentiating both sides of (46) with respect to $x$, we get

$$
\begin{gathered}
-\frac{1}{(n-m-2) !} \int_{x}^{\infty} e^{t y}[-\ln \bar{F}(y)+\ln \bar{F}(x)]^{n-m-2} \\
\cdot \frac{f(x)}{\bar{F}(x)} f(y)=f(x) H_{m}(x)+H_{m}^{\prime}(x) \bar{F}(x)
\end{gathered}
$$

or

$$
\frac{f(x)}{\bar{F}(x)}=-\frac{H_{m}^{\prime}(x)}{\left[H_{m+1}(x)-H_{m}(x)\right]}=\left(\frac{\theta}{e^{-x}+1}\right)
$$

which proves that

$$
F(x)=1-\frac{2^{\theta}}{\left(1+e^{x}\right)^{\theta}}, \quad 0 \leq x<\infty, \theta>0 .
$$

\section{Estimation}

In this section, we obtain the maximum likelihood estimators of the parameters $\theta, \mu$, and $\sigma$ of type I generalized half-logistic distribution by using the method of least squares based on the upper record values.

Let $X_{1}, X_{2}, \ldots$ be a sequence of iid random variables with $c d f F(x)$ and $p d f f(x)$ on positive support. Let $Y_{n}=$ $\max \left\{X_{1}, X_{2}, \ldots, X_{n}\right\}$ for $n \geq 1$. The observation $Y_{j}, j \geq 1$, is an upper record value of this sequence, if it is greater than all preceding observations; that is, $Y_{j}>Y_{j-1}$ for $j>1$.

Suppose we observe $n$ upper record values $X_{U(1)}$, $X_{U(2)}, \ldots, X_{U(n)}$ from a sequence of iid random variables from type I generalized half-logistic distribution with $p d f$ (3). The likelihood function based on the random sample of size $n$ is obtained from

$$
\begin{aligned}
& L(\theta, \mu, \sigma \mid x) \\
& \quad=f\left(x_{U(n)} ; \theta, \mu, \sigma\right) \prod_{i=1}^{n-1} \frac{f\left(x_{U(i)} ; \theta, \mu, \sigma\right)}{1-F\left(x_{U(i)} ; \theta, \mu, \sigma\right)} .
\end{aligned}
$$

By using (7), (51) can be rewritten as

$$
\begin{aligned}
& L(\theta, \mu, \sigma \mid x) \\
& \quad=\frac{2^{\theta} \theta^{n}}{\sigma^{n}\left(1+e^{\left(x_{U(n)}-\mu\right) / \sigma}\right)^{\theta}} \prod_{i=1}^{n} \frac{e^{\left(x_{U(i)}-\mu\right) / \sigma}}{\left(1+e^{\left(x_{U(i)}-\mu\right) / \sigma}\right)} .
\end{aligned}
$$

5.1. Maximum Likelihood Estimation. The log-likelihood function for $\theta, \mu$, and $\sigma$ is given by

$$
\begin{aligned}
L(\theta, \mu, \sigma \mid x)= & \theta \ln 2+n \ln \theta-n \ln \sigma-\frac{n \mu}{\sigma} \\
& +\frac{1}{\sigma} \sum_{i=1}^{n} x_{U(i)}-\theta \ln \left(1+e^{\left(x_{U(n)}-\mu\right) / \sigma}\right) \\
& -\sum_{i=1}^{n} \ln \left(1+e^{\left(x_{U(i)}-\mu\right) / \sigma}\right) .
\end{aligned}
$$

By using the method of least squares, the normal equations are

$$
\begin{aligned}
\frac{\partial l}{\partial \theta}= & \ln 2+\frac{n}{\theta}-\ln \left(1+e^{\left(x_{U(n)}-\mu\right) / \sigma}\right)=0 \\
\frac{\partial l}{\partial \mu}= & -\frac{n}{\sigma}+\frac{\theta e^{\left(x_{U(n)}-\mu\right) / \sigma}}{\sigma\left(1+e^{\left(x_{U(n)}-\mu\right) / \sigma}\right)} \\
& +\frac{1}{\sigma} \sum_{i=1}^{n} \frac{e^{\left(x_{U(i)}-\mu\right) / \sigma}}{\left(1+e^{\left(x_{U(i)}-\mu\right) / \sigma}\right)}=0 \\
\frac{\partial l}{\partial \sigma}= & -\frac{n}{\sigma}-\frac{1}{\sigma^{2}} \sum_{i=1}^{n} x_{U(i)}+\frac{n \mu}{\sigma^{2}} \\
& +\frac{\theta\left(x_{U(n)}-\mu\right) e^{\left(x_{U(n)}-\mu\right) / \sigma}}{\sigma^{2}\left(1+e^{\left(x_{U(n)}-\mu\right) / \sigma}\right)} \\
& +\frac{1}{\sigma^{2}} \sum_{i=1}^{n} \frac{\left(x_{U(i)}-\mu\right) e^{\left(x_{U(i)}-\mu\right) / \sigma}}{\left(1+e^{\left(x_{U(i)}-\mu\right) / \sigma}\right)}=0 .
\end{aligned}
$$

The maximum likelihood estimators $\hat{\theta}, \widehat{\mu}$, and $\widehat{\sigma}$ of the parameters $\theta, \mu$, and $\sigma$, respectively, are obtained from the above nonlinear equations and can be computed by using numerical methods.

5.2. Asymptotic Confidence Interval Estimation. In this section, we obtain the asymptotic confidence intervals for the parameters $\theta, \mu$, and $\sigma$ of type I generalized half-logistic distribution.

For obtaining confidence intervals, we define Fisher information matrix as

$$
I(\Theta)=\left(\begin{array}{ccc}
I_{\theta \theta} & I_{\theta \mu} & I_{\theta \sigma} \\
& I_{\mu \mu} & I_{\mu \sigma} \\
& & I_{\sigma \sigma}
\end{array}\right),
$$

where the elements are given in the Appendix. The expectations in the Fisher information matrix can be obtained numerically. Let $\widehat{\Theta}=(\widehat{\theta}, \widehat{\mu}, \widehat{\sigma})^{T}$ be the maximum likelihood estimate of $\Theta=(\theta, \mu, \sigma)^{T}$. Now assuming that the usual regularity conditions hold true and that the parameters are in the interior of the parameter space, but not on the boundary, we get $\sqrt{n}(\widehat{\Theta}-\Theta) \stackrel{d}{\rightarrow} N_{3}\left(\underline{0}, I^{-1}(\Theta)\right.$, where $\left.I(\Theta)\right)$ is the expected Fisher information matrix. The asymptotic behaviour is still valid if $I(\Theta)$ is replaced by the observed information matrix 
evaluated at $\widehat{\Theta}$, that is, $I(\widehat{\Theta})$. Now the multivariate normal distribution $N_{3}\left(\underline{0}, I^{-1}(\widehat{\Theta})\right)$, where the mean vector $\underline{0}=$ $(0,0,0)^{T}$, can be used to construct confidence intervals and confidence regions for the individual parameters.

The asymptotic $100(1-\varphi) \%$ two-sided confidence intervals for $\theta, \mu$, and $\sigma$ are given by

$$
\begin{aligned}
& \widehat{\theta} \pm z_{\varphi / 2} \sqrt{I_{\theta \theta}^{-1}(\widehat{\Theta})}, \\
& \widehat{\mu} \pm z_{\varphi / 2} \sqrt{I_{\mu \mu}^{-1}(\widehat{\Theta})}, \\
& \widehat{\sigma} \pm z_{\varphi / 2} \sqrt{I_{\sigma \sigma}^{-1}(\widehat{\Theta})},
\end{aligned}
$$

respectively, where $z_{\varphi / 2}$ is the upper $(\varphi / 2)$ th percentile of a standard normal distribution.

\section{Simulation Study}

In this section, we are carrying out simulation procedure for computing maximum likelihood estimates of the parameters $\theta, \mu$, and $\sigma$ of the type I generalized half-logistic distribution based on upper record values for different sample sizes $(n=$ $5,7,10,15)$. We proceed by using 1000 iterations for the true values of the parameters: $\theta=1, \mu=0.5$, and $\sigma=1$. Table 3 provides the MLEs of the three parameters and their respective mean squared errors (MSEs) in the parentheses associated with them, respectively. From the results obtained, we infer that the MLE of parameter $\theta$ is 0.5 irrespective of sample sizes and hence the MSE is 0.25 . Also we see that as the sample size increases, the estimates of the other two parameters $\mu$ and $\sigma$ tend to be closer to their true parameter values and their MSEs decrease as the sample size increases which quantifies the consistency of the estimation procedure.

\section{Real Data Analysis}

To illustrate the results of this paper, we analyze one real data set. Consider the following data which represent failure times, in minutes, for a specific type of electrical insulation in an experiment in which the insulation was subjected to a continuously increasing voltage stress (Balakrishnan and Puthenpura [5], Lawless [30]):

From this data set, we extract $n=4$ upper record values $21.8,70.7,138.6$, and 151.9. By using the method of maximum likelihood described in Section 5, we compute the maximum likelihood estimates as well as 95\% confidence intervals for the parameters $\theta, \mu$, and $\sigma$. The maximum likelihood estimates of the parameters $\theta, \mu$, and $\sigma$ are 0.1295051, 11.67251, and 4.439656, respectively. The 95\% confidence intervals for the parameters $\theta, \mu$, and $\sigma$ are $(-0.6392060,0.8982162)$, $(0.5261195,22.8188985)$, and $(-22.73694,31.61625)$, respectively.
TABLE 3: Simulation results: MLEs of $\theta, \mu$, and $\sigma$ with their respective MSEs.

\begin{tabular}{cccc}
\hline$n$ & $\widehat{\theta}$ & $\widehat{\mu}$ & $\widehat{\sigma}$ \\
\hline \multirow{2}{*}{5} & 0.5 & 1.787478 & 0.566887 \\
& $(0.25)$ & $(2.094613)$ & $(0.418088)$ \\
\hline 7 & 0.5 & 1.328763 & 0.689088 \\
& $(0.25)$ & $(1.562644)$ & $(0.273315)$ \\
\hline \multirow{2}{*}{10} & 0.5 & 1.021207 & 0.815709 \\
& $(0.25)$ & $(0.510523)$ & $(0.255344)$ \\
\hline \multirow{2}{*}{15} & 0.5 & 0.870377 & 0.960903 \\
& $(0.25)$ & $(0.496804)$ & $(0.212186)$ \\
\hline
\end{tabular}

\section{Concluding Remarks}

(i) In this study, some new explicit expressions and recurrence relations for marginal and joint moment generating functions of $k$ th upper record values from the type I generalized half-logistic distribution have been established. Further, characterization of this distribution has also been obtained on using the conditional expectation of record values. Finally, we obtain the maximum likelihood estimators of upper record values and their confidence intervals.

(ii) The recurrence relations for moments of ordered random variables are important because they reduce the amount of direct computations for moments, evaluate the higher order moments, and can be used to characterize distributions.

(iii) The recurrence relations of higher order joint moments enable us to derive single, product, triple, and quadruple moments which can be used in Edgeworth approximate inference.

(iv) In this paper, we computed moments, maximum likelihood estimates of the parameters of upper record values, and their confidence intervals to infer the main characteristics of the type I generalized halflogistic distribution.

\section{Appendix}

Consider the following:

$$
\begin{aligned}
I_{\theta \theta} & =-\frac{n}{\theta^{2}} \\
I_{\mu \mu} & =-\frac{\theta e^{\left(x_{U(n)}-\mu\right) / \sigma}}{\sigma^{2}\left(1+e^{\left(x_{U(n)}-\mu\right) / \sigma}\right)^{2}}+\frac{1}{\sigma^{2}} \sum_{i=1}^{n} \frac{e^{\left(x_{U(i)}-\mu\right) / \sigma}}{\left(1+e^{\left(x_{U(i)}-\mu\right) / \sigma}\right)^{2}}, \\
I_{\sigma \sigma} & =\frac{n}{\sigma^{2}}-\frac{2 n \mu}{\sigma^{3}} \\
& +\frac{\theta \mu e^{\left(x_{U(n)}-\mu\right) / \sigma}\left[\left(x_{U(n)}-\mu\right)+2 \sigma\left(1+e^{\left(x_{U(n)}-\mu\right) / \sigma}\right)\right]}{\sigma^{4}\left(1+e^{\left(x_{U(n)}-\mu\right) / \sigma}\right)^{2}}+\frac{2}{\sigma^{3}} \\
& \cdot \sum_{i=1}^{n} x_{U(i)}+\frac{\mu}{\sigma^{4}} \\
& \cdot \sum_{i=1}^{n} \frac{e^{\left(x_{U(i)}-\mu\right) / \sigma}\left[\left(x_{U(i)}-\mu\right)+2 \sigma\left(1+e^{\left(x_{U(i)}-\mu\right) / \sigma}\right)\right]}{\left(1+e^{\left(x_{U(i)}-\mu\right) / \sigma}\right)^{2}},
\end{aligned}
$$




$$
\begin{aligned}
I_{\theta \mu} & =\frac{e^{\left(x_{U(n)}-\mu\right) / \sigma}}{\sigma\left(1+e^{\left(x_{U(n)}-\mu\right) / \sigma}\right)}, \\
I_{\theta \sigma} & =\frac{\left(x_{U(n)}-\mu\right) e^{\left(x_{U(n)}-\mu\right) / \sigma}}{\sigma^{2}\left(1+e^{\left(x_{U(n)}-\mu\right) / \sigma}\right)}, \\
I_{\mu \sigma} & =\frac{n}{\sigma^{2}}-\frac{\theta e^{\left(x_{U(n)}-\mu\right) / \sigma}\left[1+\left(x_{U(n)}-\mu\right) / \sigma+e^{\left(x_{U(n)}-\mu\right) / \sigma}\right]}{\sigma^{2}\left(1+e^{\left(x_{U(n)}-\mu\right) / \sigma}\right)^{2}} \\
& -\frac{1}{\sigma^{2}} \\
& \cdot \sum_{i=1}^{n} \frac{e^{\left(x_{U(i)}-\mu\right) / \sigma}\left[1+\left(x_{U(i)}-\mu\right)\left(x_{U(i)}-\mu\right) / \sigma+e^{\left(x_{U(i)}-\mu\right) / \sigma}\right]}{\left(1+e^{\left(x_{U(i)}-\mu\right) / \sigma}\right)^{2}} .
\end{aligned}
$$

\section{Conflict of Interests}

The authors declare that there is no conflict of interests regarding the publication of this paper.

\section{Acknowledgment}

The authors are grateful to the anonymous reviewers for their valuable comments and suggestions in improving the paper.

\section{References}

[1] N. Balakrishnan, "Order statistics from the half logistic distribution," Journal of Statistical Computation and Simulation, vol. 20, no. 4, pp. 287-309, 1985.

[2] N. Balakrishnan and R. Aggarwala, "Relationships for moments of order statistics from the right-truncated generalized half logistic distribution," Annals of the Institute of Statistical Mathematics, vol. 48, no. 3, pp. 519-534, 1996.

[3] N. Balakrishnan and K. H. Wong, "Best linear unbiased estimation of location and scale parameters of the half-logistic distribution based on type-II censored samples," The American Journal of Mathematical and Management Sciences, vol. 14, no. 1-2, pp. 53-101, 1994.

[4] N. Balakrishnan and P. S. Chan, "Estimation for the scaled half logistic distribution under type-II censoring," Computational Statistics \& Data Analysis, vol. 13, no. 2, pp. 123-141, 1992.

[5] N. Balakrishnan and S. Puthenpura, "Best linear unbiased estimators of location and scale parameters of the half logistic distribution," Journal of Statistical Computation and Simulation, vol. 25, no. 3-4, pp. 193-204, 1986.

[6] N. Balakrishnan and K. H. T. Wong, "Approximate MLEs for the location and scale parameters of the half-logistic distribution with type-II right-censoring," IEEE Transactions on Reliability, vol. 40, no. 2, pp. 140-145, 1991.

[7] H. Torabi and F. L. Bagheri, "Estimation of parameters for an extended generalized half logistic distribution based on complete and censored data," Journal of the Iranian Statistical Society, vol. 9, no. 2, pp. 171-195, 2010.

[8] K. N. Chandler, "The distribution and frequency of record values," Journal of the Royal Statistical Society Series B: Methodological, vol. 14, pp. 220-228, 1952.

[9] W. Feller, An Introduction to Probability Theory and Its Applications, vol. 2, John Wiley and Sons, New York, NY, USA, 1966.
[10] S. I. Resnick, Extreme Values, Regular Variation and Point Processes, Springer, New York, NY, USA, 1973.

[11] M. Ahsanullah, Record Statistics, Nova Science, Commack, NY, USA, 1995.

[12] B. C. Arnold, N. Balakrishnan, and H. N. Nagaraja, A First Course in Order Statistics, John Wiley \& Sons, New York, NY, USA, 1992.

[13] B. C. Arnold, N. Balakrishnan, and H. N. Nagaraja, Records, John Wiley \& Sons, New York, NY, USA, 1998.

[14] V. B. Nevzorov, "Records," Theory of Probability \& Its Applications, vol. 32, pp. 219-251, 1987.

[15] U. Kamps, "A concept of generalized order statistics," Journal of Statistical Planning and Inference, vol. 48, no. 1, pp. 1-23, 1995.

[16] W. Dziubdziela and B. Kopocinski, "Limiting properties of the k-th record value," Applicationes Mathematicae, vol. 15, pp. 187190,1976

[17] Z. Grudzień, Characterization of distribution of time limits in record statistics as well as distributions and moments of linear record statistics from the sample of random numbers [Praca Doktorska], UMCS, Lublin, Poland, 1982.

[18] D. Kumar, "Recurrence relations for marginal and joint moment generating functions of generalized logistic distribution based on lower $K$ record values and its characterization," ProbStat Forum, vol. 5, pp. 47-53, 2012.

[19] M. Ahsanullah and M. Z. Raqab, "Recurrence relations for the moment generating functions of record values from Pareto and Gumble distributions," Stochastic Modelling Applications, vol. 2, pp. 35-48, 1999.

[20] M. Z. Raqab and M. Ahsanullah, "Relations for marginal and joint moment generating functions of record values from power function distribution," Journal of Applied Statistical Science, vol. 10 , no. 1, pp. 27-36, 2000.

[21] M. Z. Raqab and M. Ahsanullah, "On moment generating function of records from extreme value distribution," Pakistan Journal of Statistics, vol. 19, no. 1, pp. 1-13, 2003.

[22] P. Pawlas and D. Szynal, "Relations for single and product moments of th record values from exponential and Gumbel distributions," Journal of Applied Statistical Science, vol. 7, pp. 53-61, 1998.

[23] P. Pawlas and D. Szynal, "Recurrence relations for single and product moments of $k$-th record values from Pareto, generalized Pareto and Burr distributions," Communications in StatisticsTheory and Methods, vol. 28, no. 7, pp. 1699-1709, 1999.

[24] P. Pawlas and D. Szynal, "Recurrence relations for single and product moments of $k$-th record values from Weibull distributions, and a characterization," Journal of Applied Statistical Science, vol. 10, no. 1, pp. 17-25, 2000.

[25] K. S. Sultan, "Record values from the modified Weibull distribution and applications," International Mathematical Forum, vol. 2, no. 41, pp. 2045-2054, 2007.

[26] D. Kumar, "Relations for moments of $K$-th lower record values from exponentiated log-logistic distribution and a characterization," International Journal of Mathematical Archive, vol. 6, pp. $1-7,2011$.

[27] D. Kumar and A. Kulshrestha, "Recurrence relations for moments of kth record values from generalized Pareto distribution and a characterization," Journal of Statistics Application in Probability, vol. 13, pp. 75-82, 2013.

[28] A. K. Olapade, "The type I generalized half logistic distribution," Journal of the Iranian Statistical Society, vol. 13, no. 1, pp. 69-82, 2014. 
[29] G. D. Lin, "On a moment problem," Tohoku Mathematical Journal, vol. 38, no. 4, pp. 595-598, 1986.

[30] J. F. Lawless, Statistical Models and Methods for Lifetime Data, John Wiley \& Sons, New York, NY, USA, 1982. 


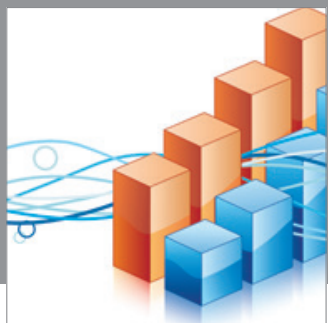

Advances in

Operations Research

mansans

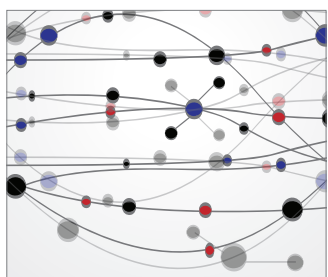

The Scientific World Journal
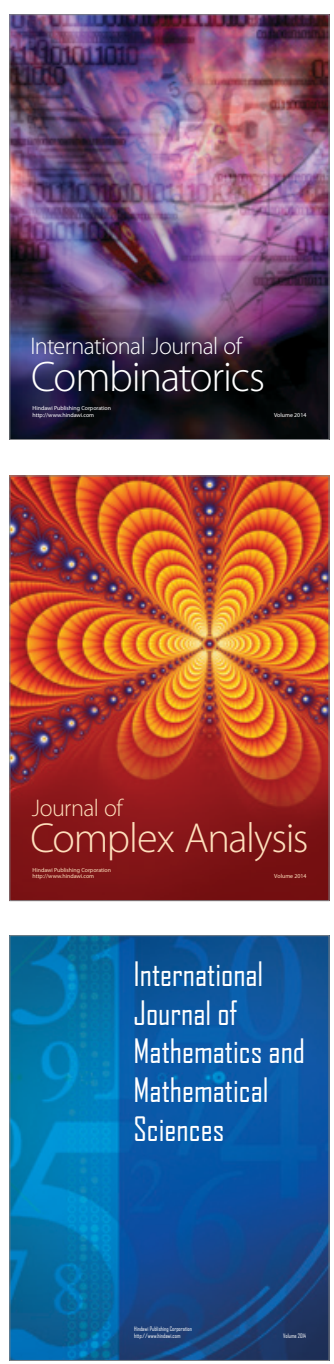
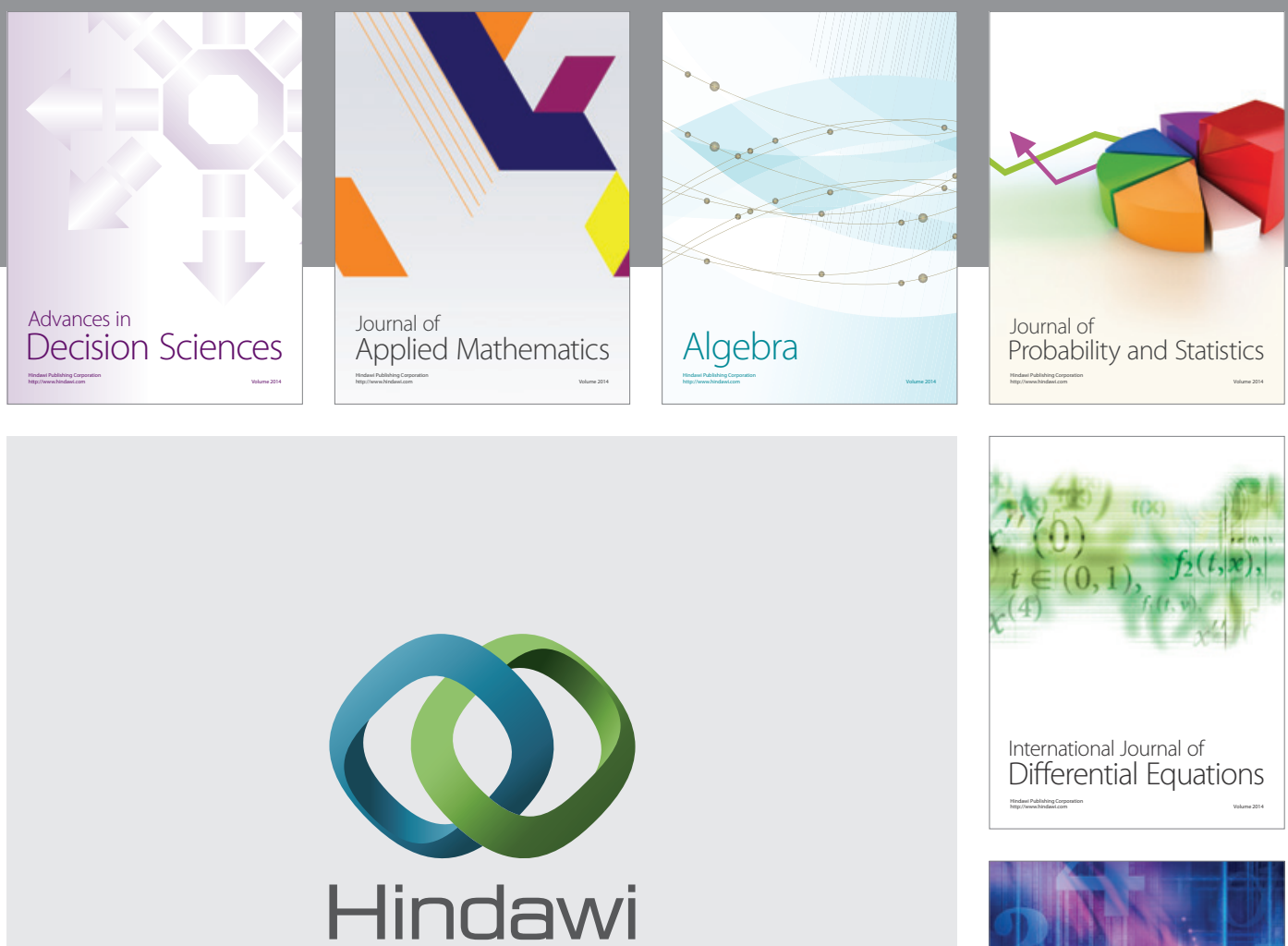

Submit your manuscripts at http://www.hindawi.com
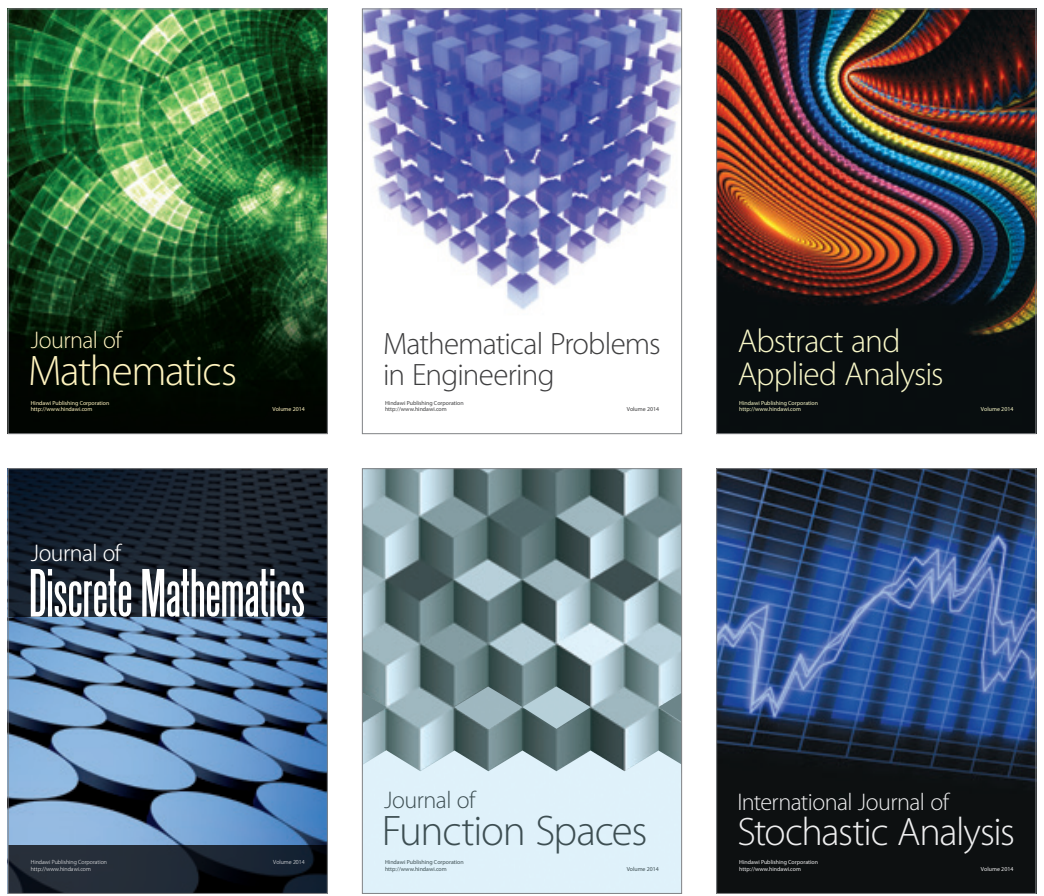

Journal of

Function Spaces

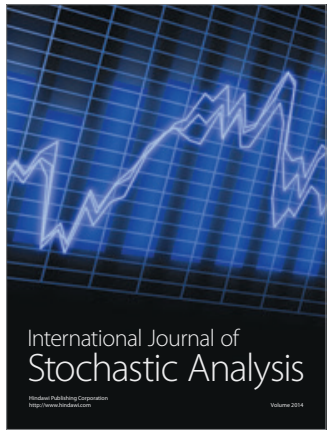

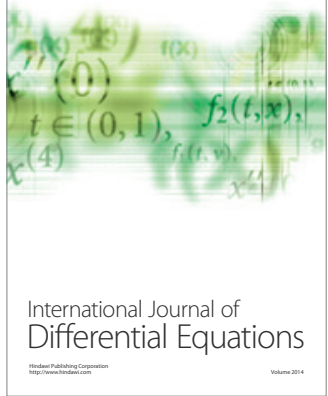
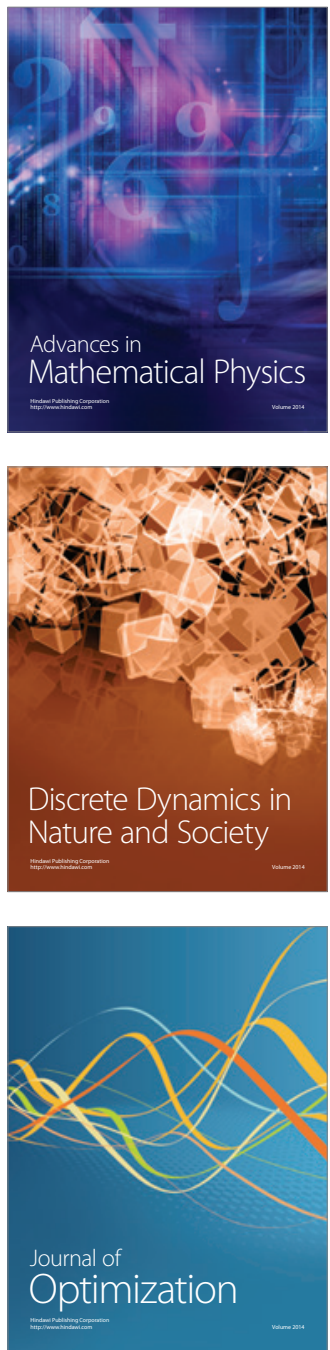